\title{
Energy Eigenvalue of Hulthen Potential Using Nikiforov-Uvarov and Asymptotic Iterative Method of Hydrogen and Hydrogen-Like Atom
}

\author{
Bhishma Karki ${ }^{1,2}$, Saddam Husain Dhobi ${ }^{3,4,5}$, Kishori Yadav ${ }^{3,5}$, Jeevan Jyoti Nakarmi,5 \\ ${ }^{1}$ Department of Physics, Tri-Chandra Multiple Campus, Tribhuvan University, Kathmandu, Nepal \\ ${ }^{2}$ National Research Council, New Baneshwor, Kathmandu, Nepal \\ ${ }^{3}$ Department of Physics, Patan Multiple Campus, Tribhuvan University, Lalitpur, Nepal \\ ${ }^{4}$ Robotics Academy of Nepal, Lalitpur, Nepal \\ ${ }^{5}$ Innovative Ghar Nepal, Lalitpur, Nepal \\ Email: *magnum.photon@gmail.com
}

How to cite this paper: Karki, B., Dhobi, S.H., Yadav, K. and Nakarmi, J.J. (2022) Energy Eigenvalue of Hulthen Potential Using Nikiforov-Uvarov and Asymptotic Iterative Method of Hydrogen and Hydrogen-Like Atom. Open Journal of Microphysics, 12, 31-46.

https://doi.org/10.4236/ojm.2022.121002

Received: August 15, 2021

Accepted: February 19, 2022

Published: February 22, 2022

Copyright $\odot 2022$ by author(s) and Scientific Research Publishing Inc. This work is licensed under the Creative Commons Attribution International License (CC BY 4.0).

http://creativecommons.org/licenses/by/4.0/

\begin{abstract}
The objective of this work is to calculate and compare the energy eigenvalue of Hulthen Potential using the NU method and AIM method. Using these two methods the energy eigenvalue calculated from the NU method is less than $\frac{\delta}{2}$ AIM method. Moreover, the energy eigenvalue calculated from both methods is charge independent and only depends upon the quantum numbers and screening parameters, while the third term of energy eigenvalue calculated using the NU method is only dependent on screening parameters.
\end{abstract}

\section{Keywords}

Hulthen Potential, NU and AI Method, Schrodinger Equation, Quantum Numbers

\section{Introduction}

Schrödinger's wave equation gives exact solutions only for an atom with one electron example like $\mathrm{H}, \mathrm{He}^{+}, \mathrm{Li}^{2+}, \mathrm{Be}^{3+}, \mathrm{B}^{4+}, \mathrm{C}^{5+}$, etc. This one-electron system is often described as hydrogenic and time-independent Schrödinger equation expressed as,

$$
H \psi=E \psi
$$

The equation is also applied to electrons traveling at non-relativistic speeds and in spherical polar coordinates (like hydrogen) system, the wave function 
$\psi=\psi(r, \theta, \phi)$ with Laplacian operator and separation variable the equation become,

$$
\begin{aligned}
& \frac{1}{r^{2}} \frac{\partial}{\partial r}\left(r^{2} \frac{\partial}{\partial r}\right) R(r)+R(r) \frac{1}{r^{2} \sin \theta} \frac{\partial}{\partial \theta}\left(\sin \theta \frac{\partial}{\partial \theta}\right) \\
& +R(r) \frac{1}{r^{2} \sin ^{2} \theta}\left(\frac{\partial^{2}}{\partial \phi^{2}}\right)-\frac{2 m}{\hbar^{2}}[V(r)-E] R(r)=0
\end{aligned}
$$

The radial part of SE from (2) is obtained [1] as,

$$
\frac{1}{r^{2}} \frac{\partial}{\partial r}\left(r^{2} \frac{\partial R(r)}{\partial r}\right)+\left(\frac{2 m}{\hbar^{2}}[E+V]-\frac{l(l+1)}{r^{2}}\right) R(r)=0
$$

AIM can be used to find exact analytical solutions to the Schrödinger equation and the corresponding eigenvalue, as well as to solve differential equations important in quantum mechanics. This method has been successfully applied to many anharmonic oscillators. AIM is a technique for solving analytically and approximately linear second-order differential equations, particularly eigenvalue problems, which occur frequently in theoretical and mathematical physics. The $\mathrm{NU}$ method is also used to solve the Schrodinger equation and has a D-module theory application to sort the eigenvalue problem. Furthermore, NU is used as an alternative treatment for calculations performed within the framework of the Nikiforov-Uvarov method, which eliminates a flaw in the original theory and avoids some difficulties in solving the Schrödinger equation. There has recently been renewed interest in solving simple quantum mechanical systems using the NU method. This algebraic technique is based on solving second-order linear differential equations, which have been successfully used to solve the Schrödinger, Dirac, Klein-Gordon, and Duffin-Kemmer-Petiau wave equations in the presence of well-known central and non-central potentials.

\section{Literature Review}

The Hulthen potential (Hulthen, 1942) relation is given by Hulthen as

$$
V(r)=-\frac{z}{a} \frac{\exp \left(-\frac{r}{a}\right)}{1-\exp \left(-\frac{r}{a}\right)}
$$

With screening parameters $\delta=$ ant which is identified with the atomic number $z$, and $r$ is distance. This potential is a short-range potential that behaves like a Coulomb potential for small values of $r$ and decreases exponentially for large values of $r$. The energy eigenvalue is strongly bounded and an increase in rotational quantum number $I$ makes energy become more attractive that the energy becomes more negative with increasing screening parameter $\delta$ [2]. The energy of Hulthen Potential decreases with an increase in a quantum state and also shows the energies apart from decreasing with an increase in screening parameter $\delta$ [3]. The Hulthen potential exhibits an exponential decline for $r \gg a$ and behaves like $1 / r$ for $0 \ll r \ll a$ is shown in figure [4] [5] [6]. 
For the solution of energy eigenvalue of Hulthen Potential, the considered method in this work is NU and AIM. The other potentials like Hulthen, Poschl-Teller, Eckart, Coulomb, Hylleraas, Pseudoharmonic, and Scarf II potentials energy eigenvalue were also calculated using this method. Moreover, other methods like Supersymmetric Quantum Mechanics Approach, Formular Method, and Exact Quantisation are also used to determine the energy eigenvalue [7]-[12]. NU method is described in terms of the hypergeometric-type second-order differential equations and given the solution by Szego in 1934 with an exact solution of the SE for certain kinds of potentials,

$$
y^{\prime \prime}+p(t) y^{\prime}+q(t) y=g(t)
$$

If $g(t)=0$, then Equation (5) is said to be a homogenous equation otherwise Equation (5) is said to be nonhomogeneous reported by Nikiforov \& Uvarov in 1988 [13]. The energy eigenvalues are obtained from the quantization condition [14] as

$$
\delta_{n}(x)=\lambda_{n}(x) s_{n-1}(x)-\lambda_{n-1}(x) s_{n}(x)=0, n=1,2,3, \cdots
$$

where, $\lambda_{n}(x)=\lambda_{n-1}^{\prime}(x)+s_{n-1}(x)+\lambda_{0}(x) \lambda_{n-1}(x)$, and $s_{n}(x)=s_{n-1}^{\prime}(x)+s_{0}(x) \lambda_{n-1}(x)$.

The deformation effects on the energy spectrum of nanoscale superlattice by changing the interatomic distances as well as varying the width and height of the potential barrier have been studied theoretically. The potential deformation has been estimated, and it has been demonstrated that the deformation potential varies depending on the edge of the forbidden band. The dependence of the effective mass on energy has also been investigated. The advancement of nanotechnology has increased the demand for atomic-sized films. New low-dimensional nanostructure graphene, for example, promises to be a key component of future nanoelectronics. Several technological methods for producing graphene films on flexible solid-state substrates have already been developed. The current state of research on strain-sensing properties of semiconductor films is a result of the development of strain-resistive sensors with semiconductor sensing elements that have high sensitivity, reliability, small dimensions, and high-technology manufacturing, among other things [15].

The streaming potential coefficient and zeta potential of two consolidated samples saturated with four monovalent electrolytes at various electrolyte concentrations were measured. For all electrolytes, the experimental results show that the streaming potential coefficient and zeta potential magnitude both decrease with increasing electrolyte concentration. It is also demonstrated that the streaming potential coefficient for a given sample is dependent on the type of electrolytes. Furthermore, the variation of the zeta potential with electrolyte types is reported and qualitatively explained. The empirical expressions between the streaming potential coefficient, the zeta potential, and electrolyte concentration are also obtained from experimental data on the streaming potential coefficient and the zeta potential [16]. 
Using the Extended Transformation (ET) method, ESPs of the Position-Dependent Mass (PDM) Schrödinger equation are generated from the Hulthen Potential (parent system). The method starts with a Co-ordinate Transformation (CT) and then moves on to a Functional Transformation (FT) of the wave function. The parent system's mass function is transformed into the generated system's mass function. There are two new ESPs created. For newly generated potentials (systems), explicit expressions of mass functions, energy eigenvalues, and corresponding wave functions are derived. The method of system-specific regrouping is also discussed [17].

\section{Materials and Method}

Hulthen potential is also well-known for describing the plasma-screening effect on the continuum electron capture rate in the solar core region reported by Carlson and Havens in 1983, which is similar to Equation (4). With $z=V_{0}=4 e^{2}$ and $\delta=\frac{2}{R}$ for the Hulthen potential parameters, where $R$ is the screening radius and the screening radius $R$ has a range between $0.3 a_{0} \leq R \leq 1.25 a_{0}$ with the Bohr radius $a_{0}=\hbar^{2} /\left(m_{e} e^{2}\right)=0.529 \AA$ [18].

\subsection{Shifting Screening Parameters}

The eigenvalues were obtained numerically for arbitrary quantum numbers $n$ and 1 with the potential parameter $\delta=0.025 \mathrm{fm}^{-1}, 0.050 \mathrm{fm}^{-1}$ and $0.075 \mathrm{fm}^{-1}$. The shifted Hulthén Potential is dominant when the screening parameter tends to zero ( $\delta \rightarrow 0 \mathrm{fm}^{-1}$ ) which is called the low screening regime with constant shifting. The centrifugal term with approximation $\frac{1}{r^{2}} \approx \frac{\delta^{2} \mathrm{e}^{-\delta r}}{\left(1-\mathrm{e}^{-\delta r}\right)^{2}}$ of Hulthen Potential with distance separation is visualized in Figure 1 given below.

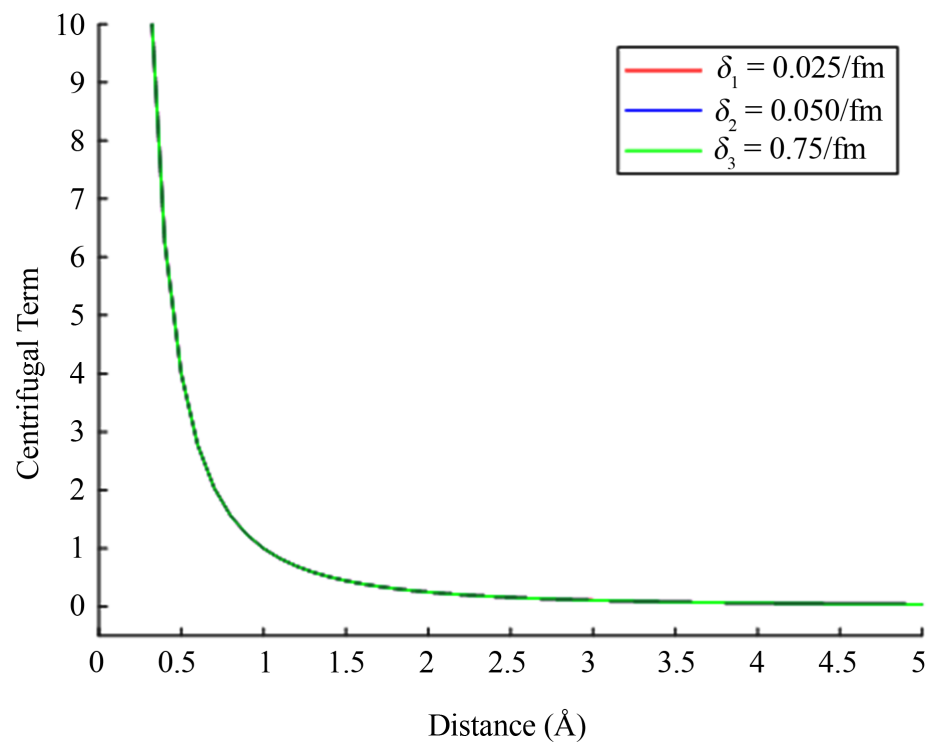

Figure 1. Centrifugal term vs distance. 
The approximation is valid only for small screening parameter $\delta$ but has an error for large in the calculation of energy eigenvalues. This approximation method solution was proposed by Greene and Aldrich in 1976 with the good agreement and later the number of authors used with the good agreement more detail in ref [19]. The nature of Hulthen potential with distance at different values of screening parameters is given below in Figure 2.

Hulthen potential has a negative value in a very small value of $r$, while the Hulthen potential inclines to be constant for a higher value of $r$ [20]. The radial Schrodinger equation for the Hulthen potential can be solved analytically only for s-states $(l=0)$. Analytically, the generalized Hulthen potential for non-zero angular momentum states given by Gonul, et al., Greene and Aldrich, and Simsek and Egrifes as

$$
V_{\text {eff }}(r)=-Z e^{2} \delta \frac{\mathrm{e}^{-\delta r}}{1-\mathrm{e}^{-\delta r}}+\frac{l(l+1) \hbar^{2} \delta^{2}}{2 m} \frac{\mathrm{e}^{-\delta r}}{\left(1-\mathrm{e}^{-\delta r}\right)^{2}}
$$

It is of much concern to see that for small values of $\delta r$, Equation (7) approximates to the effective exponential-type screened Coulomb potential as $\left(-Z e^{2} \frac{\mathrm{e}^{-\delta r}}{r}+\frac{l(l+1) \hbar^{2}}{2 m r^{2}}\right)$. In the case of Coulomb potential, small values of $r$, the Hulthen potential, behaves like a screened Coulomb potential whereas for large values of $r$ it decreases exponentially so its capacity for the bound state is smaller than the Coulomb potential [21].

The important quantity of interest for the Hulthen potential is screening parameter $\delta$, which binding energy of the level in question becomes zero and is given in atomic [19] [20] units as, $\delta=\frac{2}{(n+l+1)^{2}}$. The value of screening

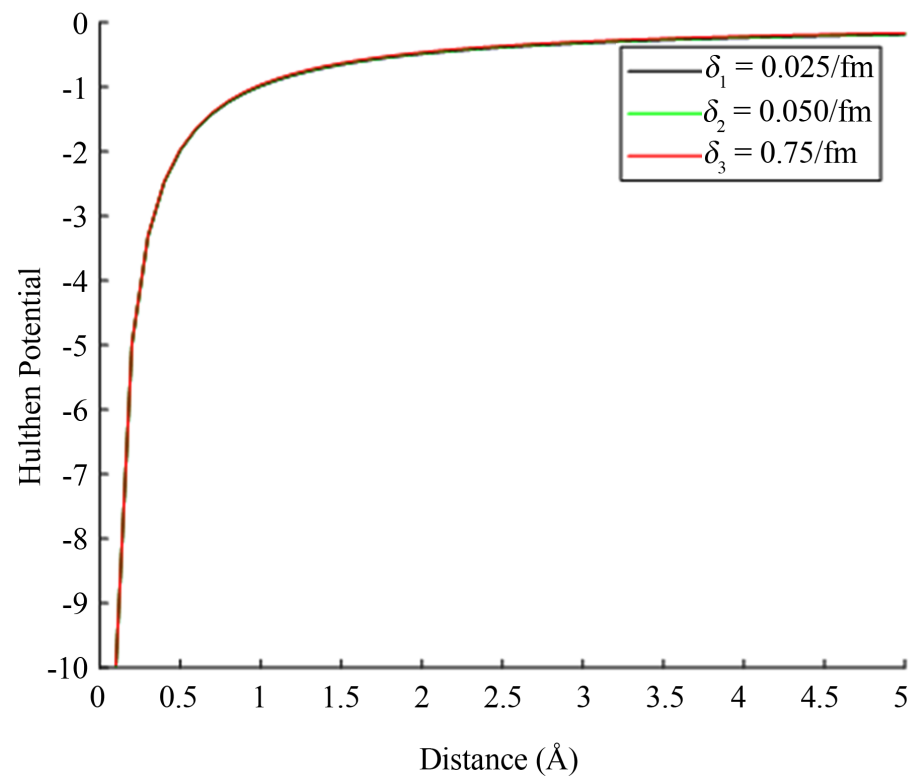

Figure 2. Hulthen potential with distance for different screening parameters. 
potential is 0.025 for low screening and 0.15 for high screening and the simple solution, in hyperbolic trigonometric term is given as [22],

$$
U(r)=\frac{U_{H} \delta}{2}(1-\operatorname{coth} \delta r)
$$

The Hulthen potential has a negative value in a very small value of $r$, while the Hulthen potential inclines to be constant for a higher value of $r$. The analytical solution of SE with $l=0$ and $l \neq 0$ for some physical potentials has been addressed by many researchers.

\subsection{Asymptotic Iteration Method}

An asymptotic iteration method for solving second-order homogeneous linear differential equations of form $y^{\prime \prime}=\lambda_{0}(x) y^{\prime}+s_{0}(x) y$ is introduced, where $\lambda_{0}(x) \neq 0$ and $s_{0}(x)$ are $C_{\infty}$ functions (Ciftei et al., 2003). Recently, some complex potentials like $V(x)=i x^{3}, V(x)=i x^{3}+i \alpha x$ and $V(x)=x^{4}+i \alpha x$ have been studied in the literature and AIM numerically calculated the Eigen energies of the new complex potential. The obtained numerical results are compared with those obtained by using the WKB, EMM, and MRF methods and found to be in excellent agreement [23]. Moreover, linear second-order differential equations with rational coefficients and explicit bases of solutions with classical a harmonic oscillator potential $V(x)=x^{2}+A x^{4}, A \geq 0$ [24].

\section{Calculation of Energy Eigen Value of Hulthen Potential with NU Method}

The radial part of SE for Hulthen Potential from Equation (3) can be obtained as

$$
R_{n l}^{\prime \prime}(r)+\frac{2}{r} R_{n l}^{\prime}(r)+\frac{2 \mu}{\hbar^{2}}\left[E+Z \delta e^{2} \frac{\mathrm{e}^{-\delta r}}{1-\mathrm{e}^{-\delta r}}-\frac{l(l+1) \hbar^{2}}{2 \mu r^{2}}\right] R_{n l}(r)=0
$$

This equation generates two cases $(l \neq 0$, and $l=0)$ with Hunthel potential being $V(r)=-Z \delta e^{2} \frac{\mathrm{e}^{-\delta r}}{1-\mathrm{e}^{-\delta r}}$. This potential of the form is defined and used by different authors like Agboola: 2009 \& 2010; Saad: 2007 and Hulthen: 1942 \& 1957 for atomic phenomena [25]. To obtain the solution of Equation (9), let us define $R_{n l}(r)=\frac{U_{n l}(r)}{r}$ and solve the equation for $\mathrm{NU}$ an obtained relation is as,

$$
U_{n l}^{\prime \prime}(r)+\left[\frac{2 \mu}{\hbar^{2}} E+\frac{2 \mu}{\hbar^{2}} Z e^{2} \delta \frac{\mathrm{e}^{-\delta r}}{1-\mathrm{e}^{-\delta r}}-\frac{l(l+1)}{r^{2}}\right] U_{n l}(r)=0
$$

On applying an approximation method on the centrifugal term used by Agboola: 2009 and Oiang et al:: 2007 Equation (10) and solve on assuming $z \rightarrow \mathrm{e}^{-\delta r}$ and $U_{n l}(r) \rightarrow \psi_{n l}(z)$ the obtained relation with $l(l+1)=\gamma$ is

$$
\psi_{n l}^{\prime \prime}(z)+\frac{1}{z} \psi_{n l}^{\prime}(z)+\left[\frac{2 \mu E}{\hbar^{2} \delta^{2} z^{2}}+\frac{2 \mu Z e^{2}}{\hbar^{2} \delta} \frac{1}{z(1-z)}-\frac{\gamma}{z(1-z)^{2}}\right] \psi_{n l}(z)=0
$$

Let $\alpha=-\frac{2 \mu E}{\hbar^{2} \delta^{2}}$ and $\beta=\frac{2 \mu z e^{2}}{\hbar^{2} \delta}$ then Equation (11) becomes 


$$
\begin{aligned}
& \psi_{n l}^{\prime \prime}(z)+\frac{1-z}{z(1-z)} \psi_{n l}^{\prime}(z) \\
& +\frac{1}{z^{2}(1-z)^{2}}\left[-(\alpha+\beta) z^{2}+(2 \alpha+\beta-\gamma) z-\alpha\right] \psi_{n l}(z)
\end{aligned}
$$

On comparing Equation (12) with the NU equation given by Nikiforov-Uvarov in 1988 one can obtain

$$
\begin{gathered}
\tilde{\sigma}(z)=-(\alpha+\beta) z^{2}+(2 \alpha+\beta-\gamma) z-\alpha \\
\tilde{\tau}(z)=1-z \\
\sigma(z)=z(1-z)
\end{gathered}
$$

Let us define a function $\pi(z)$ and parameters $\lambda$ which is used to solve the NU method [26] as

$$
\pi(z)=\frac{\sigma^{\prime}(z)-\tilde{\tau}(z)}{2} \pm \sqrt{\left(\frac{\sigma^{\prime}(z)-\tilde{\tau}(z)}{2}\right)^{2}-\tilde{\sigma}(z)+k \sigma(z)} \text {, and } \lambda=k+\pi^{\prime}(z)
$$

Now to obtain the root, a quadratic equation solution is taken and the above equation become,

$$
\pi(z)=\frac{-z}{2} \pm \frac{1}{2} \sqrt{[1+4(\alpha+\beta-k)] z^{2}-4(2 \alpha+\beta-\gamma-k) z+4 \alpha}
$$

To obtain the root, setting the square root term equal to zero (13) and solving the obtained root $(k)$ for the NU equation is $k_{ \pm}=\beta-\gamma \pm \sqrt{\alpha(1+4 \gamma)}$.

The visualization of the nature of the root with the atomic unit $(\hbar=m=e=1$, $l=1$, and $E=10 \mathrm{MeV}$ ) is shown below in Figure 3 . The root value is very close to zero with increasing screening parameters and remains constant. For the small value of screening constant $\left(<1 \mathrm{fm}^{-1}\right)$ the root is extremely high.

Since the NU method has two roots (positive and negative), for our choice positive root is selected, and hence Equation (13) becomes

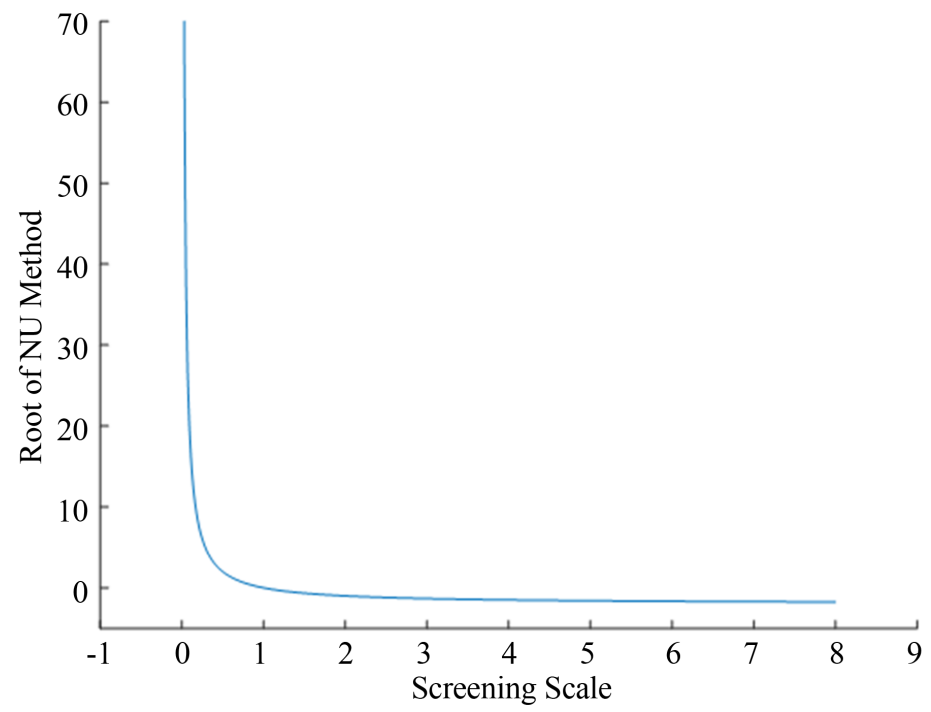

Figure 3. Root of NU method with screening parameters or scales. 


$$
\pi_{+}(z)=\frac{-z}{2}+\frac{1}{2}(\{(2 \sqrt{\alpha})-\sqrt{(1+4 \gamma)}\} z-2 \sqrt{\alpha}) \text { for } k_{+}=\beta-\gamma+\sqrt{\alpha(1+4 \gamma)}
$$

Similarly, for negative root Equation (13) also represented as

$$
\pi_{-}(z)=\frac{-z}{2}-\frac{1}{2}(\{(2 \sqrt{\alpha})+\sqrt{(1+4 \gamma)}\} z-2 \sqrt{\alpha}) \text { for } k_{-}=\beta-\gamma-\sqrt{\alpha(1+4 \gamma)}
$$

To the obtained physical solution, first, ensure that consider the polynomial is polynomial and verify the revelation $\tau(z)=\tilde{\tau}(z)+2 \pi_{-}(s)$. To satisfy the polynomial condition $\left(\tau^{\prime}(z)<0\right)$, one selects any Equation $(14$ 's), therefore a condition for polynomial becomes, $\tau^{\prime}(z)=-2-(\{(2 \sqrt{\alpha})+\sqrt{(1+4 \gamma)}\})<0$. This means consider polynomial is polynomial. Now to obtain the value of NU parameters $\lambda=k+\pi^{\prime}(z)$ for $k_{-}$the NU parameters become $\lambda=k_{-}+\pi^{\prime}(z)$.

Since $\lambda=\lambda_{n}$ where $\lambda_{n}=-n \tau^{\prime}(z)-n(n-1) \frac{\sigma^{\prime \prime}(z)}{2}$ for more detail of this relation and equalities, one can see (Sever et al. 2018). Now consider this relation and substituting the value the relation become

$k_{-}+\pi_{-}^{\prime}(z)=-n \tau^{\prime}(z)-n(n-1) \frac{\sigma^{\prime \prime}(z)}{2}$ and substituting the value and continues solving for energy,

$$
E_{n l}=-\frac{\hbar^{2}}{2 \mu}\left[\frac{\mu Z z}{\hbar^{2}(1+l+n)}-\frac{\delta}{2}(1+l+n)-\frac{\delta}{2}\right]^{2}
$$

This Equation (15) gives energy eigenvalue for Hulthen potential for quantum numbers $n$ and $l$. In atomic unit $z=1$ Equation (15) become

$$
E_{n l}=-\frac{1}{2}\left[\frac{1}{1+l+n}-\frac{\delta}{2}(1+l+n)-\frac{\delta}{2}\right]^{2}
$$

This equation is more similar to Equation (32) obtained by Ikhdair and Sever in 2008, the result obtained in Equation (16) in this work has one addition term $\left(-\frac{\delta}{2}\right)$. This term with screening parameters gives more precious to find the energy eigenvalue for Hulthen potential. The screening parameters is given [19] are related to the quantum number and angular momentum as above mention.

The nature of the screening parameters with a quantum number is given in below in Figure 4 and as the quantum number increase, the screening parameter value or screening scale decreases. In the case of azimuthal quantum number similar behavior are observed that is low azimuthal quantum number has high screening and high have low screening.

\subsection{Development of Quantization Condition for Eigen Energy Value Using AIM}

AIM is another method proposed to obtain the exact solution of a second-order differential equation with a general solution. On introducing an asymptotic 


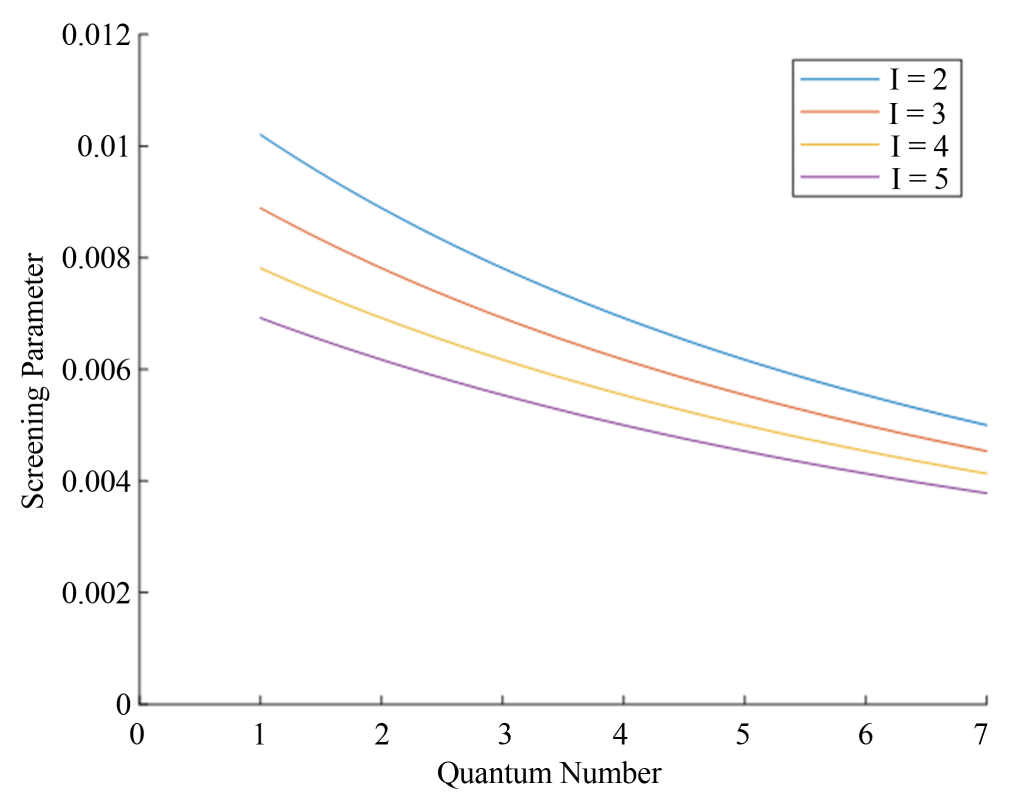

Figure 4. Screening parameters with quantum numbers.

aspect of the iteration method for sufficiently larger value of $n$ as $\frac{s_{n}}{\lambda_{n}}=\frac{s_{n-1}}{\lambda_{n-1}}=\alpha$ and the reduced form $\frac{y^{(n+2)}(x)}{y^{(n+1)}(x)}=\frac{\lambda_{n}}{\lambda_{n-1}}$ with a general solution

$$
y(x)=\exp \left[-\int \alpha(x) \mathrm{d} x\left\{C^{\prime}+C \int \exp \left(\int\left[\lambda_{0}(x)+2 \alpha(x)\right] \mathrm{d} x\right) \mathrm{d} x\right\}\right]
$$

where $C^{\prime}$ the new integration constant and the energy eigenvalue is obtained from the roots using the quantization condition of Equation (6).

Calculation of Energy Eigen Value of Hulthen Potential with AIM Method The Hulthen potential $V_{H}(r)=-z e^{2} \delta \frac{\mathrm{e}^{-\delta r}}{1-\mathrm{e}^{-\delta r}}+\frac{l(l+1) \hbar^{2}}{2 m r^{2}}$, with $\frac{l(l+1) \hbar^{2}}{2 m r^{2}}=V_{l}$, is known as the centrifugal term. The visualization of a centrifugal term with $l=1$ is shown in Figure 5.

On repeating the process up (9), with $\frac{1}{r^{2}} \approx \frac{\delta^{2} \mathrm{e}^{-\delta r}}{\left(1-\mathrm{e}^{-\delta r}\right)^{2}}, R_{n l}(r)=\frac{R_{n l}(x)}{\delta}$ and $\delta r=x$, one obtained an equation,

$$
R_{n l}^{\prime \prime}(x)+\left(\frac{2 m E}{\hbar^{2} \delta^{2}}+\frac{2 m z e^{2}}{\hbar^{2} \delta} \frac{\mathrm{e}^{-x}}{1-\mathrm{e}^{-x}}-l(l+1) \frac{\mathrm{e}^{-x}}{\left(1-\mathrm{e}^{-x}\right)^{2}}\right) R_{n l}(x)=0
$$

Let $\alpha^{2}=-\frac{2 m E}{\hbar^{2} \delta^{2}}, \quad \beta^{2}=\frac{2 m e^{2} Z}{\hbar^{2} \delta}$ and transformation the equation with $z \rightarrow \mathrm{e}^{-x} ?$ and $R_{n l}(x) \rightarrow \psi_{n l}(z)$ the obtained equation become,

$$
\psi_{n l}^{\prime \prime}(z)+\frac{1}{z} \psi_{n l}^{\prime}(z)+\left(-\frac{\alpha^{2}}{z^{2}}+\frac{\beta}{z(1-z)}-\frac{l(l+1)}{z(1-z)^{2}}\right) \psi_{n l}(z)=0
$$




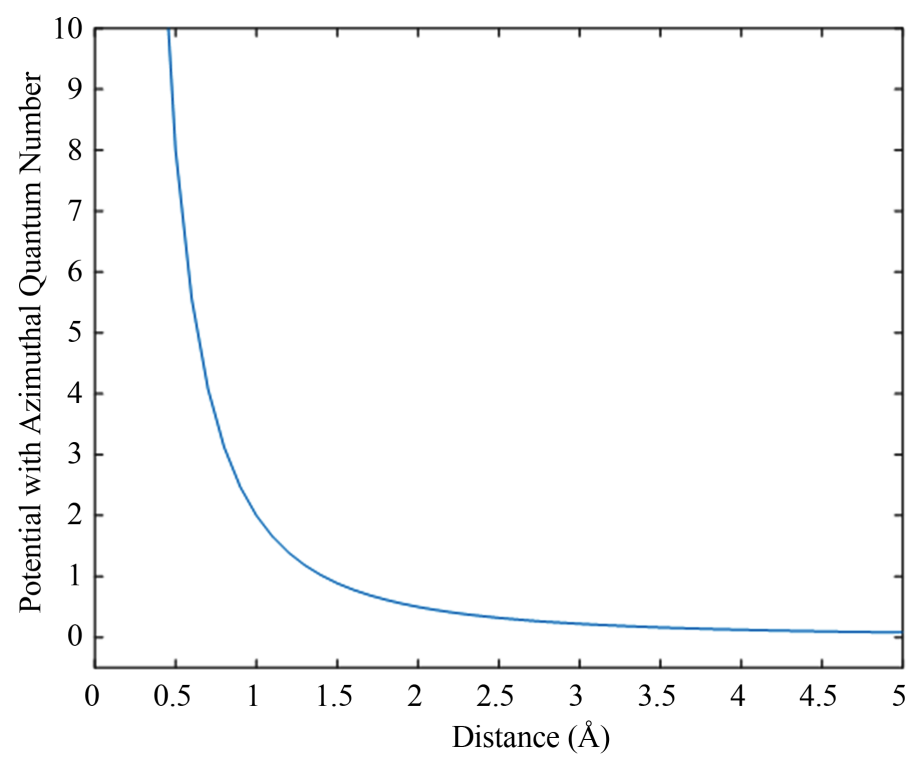

Figure 5. Centrifugal potential with azimuthal quantum number.

To solve this equation using AIM, this equation is transformed to AIM equation by proposing the reasonable physical wave function as, $\psi_{n l}(z)=z^{\alpha}(1-z)^{l+1} f_{n l}(z)$ with this wave function Equation (19) become second-order homogenous liner differentiating equation,

$$
\begin{aligned}
\frac{\mathrm{d}^{2} f_{n l}(z)}{\mathrm{d} z^{2}}= & {\left[\frac{(2 \alpha+2 l+3) z-(2 \alpha+1)}{z(1-z)}\right] \frac{\mathrm{d} f_{n l}(z)}{\mathrm{d} z} } \\
& +\left[\frac{(2 \alpha+l+2) l+2 \alpha-\beta^{2}+1}{z(1-z)}\right] f_{n l}(z)
\end{aligned}
$$

Now comparison this equation with the standard equation of AIM, the value of $\lambda_{0}(z)$ and $s_{0}(z)$ become,

$$
\begin{aligned}
& \lambda_{0}(z)=\left[\frac{(2 \alpha+2 l+3) z-(2 \alpha+1)}{z(1-z)}\right] \\
& s_{0}(z)=\left[\frac{(2 \alpha+l+2) l+2 \alpha-\beta^{2}+1}{z(1-z)}\right]
\end{aligned}
$$

Now to calculate $\lambda_{n}(z)$ and $s_{n}(z)$, we have, $\lambda_{n}(x)=\lambda_{n-1}^{\prime}+\lambda_{n-1} \lambda_{0}+s_{n-1}$. Therefore, for $n=1$,

$$
\begin{aligned}
\lambda_{1}(z)= & \frac{2+6 \alpha-7 z-2 z l-\beta^{2} z+12 z^{2} l-18 \alpha z-6 \alpha z l}{z^{2}(z-1)^{2}} \\
& +\frac{12 \alpha z^{2}+11 z^{2}+4 \alpha^{2}+l^{2} z+\beta^{2} z^{2}+4 \alpha^{2} z^{2}-8 \alpha^{2} z+6 \alpha z^{2} l+3 l^{2} z^{2}}{z^{2}(z-1)^{2}}
\end{aligned}
$$

And for $n=1$,

$$
s_{1}(z)=\frac{\left(2 l+2 \alpha+2 \alpha l+l^{2}+1-\beta^{2}\right)(-2+5 z-2 \alpha+2 \alpha z+2 \alpha l)}{z^{2}(z-1)^{2}}
$$


Similar for $\lambda_{2}, \lambda_{3}, \cdots$ and $s_{2}, s_{3}, \cdots$ are calculated for $n=2,3, \cdots$.

Now applying the quantization condition from Equation (6), for $n=1,2$, and 3 , the energy equation is $\alpha_{0}=\frac{\beta^{2}-l^{2}-2 l-1}{2(l+1)}, \alpha_{1}=\frac{\beta^{2}-4 l^{2}-4 l-4}{2(l+1)}$, and $\alpha_{2}=\frac{\beta^{2}-l^{2}-6 l-9}{2(l+1) ?}$, etc. Therefore the generalized expression of energy eigenvalue for $n=1,2,3, \cdots$ written as

$$
\alpha_{n l}=\left(\frac{\beta^{2}-(n+l+1)^{2}}{2(n+l+1)}\right), n, l=0,1,2,3, \cdots
$$

Substituting the value of $\alpha^{2}=-\frac{2 m E}{\hbar^{2} \delta^{2}}$ and $\beta^{2}=\frac{2 m e^{2} Z}{\hbar^{2} \delta}$ and applying $\alpha=\alpha_{n l}$ [27], the energy eigenvalue for $n^{\text {th }}$ is given as,

$$
E_{n l}=-\frac{\hbar^{2}}{2 m}\left(\frac{m e^{2} Z}{(n+l+1) \hbar^{2}}-\frac{(n+l+1) \delta}{2}\right)^{2}
$$

For atomic unit, $(\hbar=m=e=1)$ and $Z=1$ then this equation become

$$
E_{n l}=-\frac{1}{2}\left(\frac{1}{(n+l+1)}-\frac{(n+l+1) \delta}{2}\right)^{2}
$$

This equation is the energy eigenvalue for Hulthen potential which is charge independent and quantum numbers and screening parameters dependent

\section{Results and Discussion}

\subsection{Energy Eigenvalue of Hulthen Potential Using NU Method with Screening Parameters}

The representation Figure 6 below shows the nature of the energy eigenvalue of

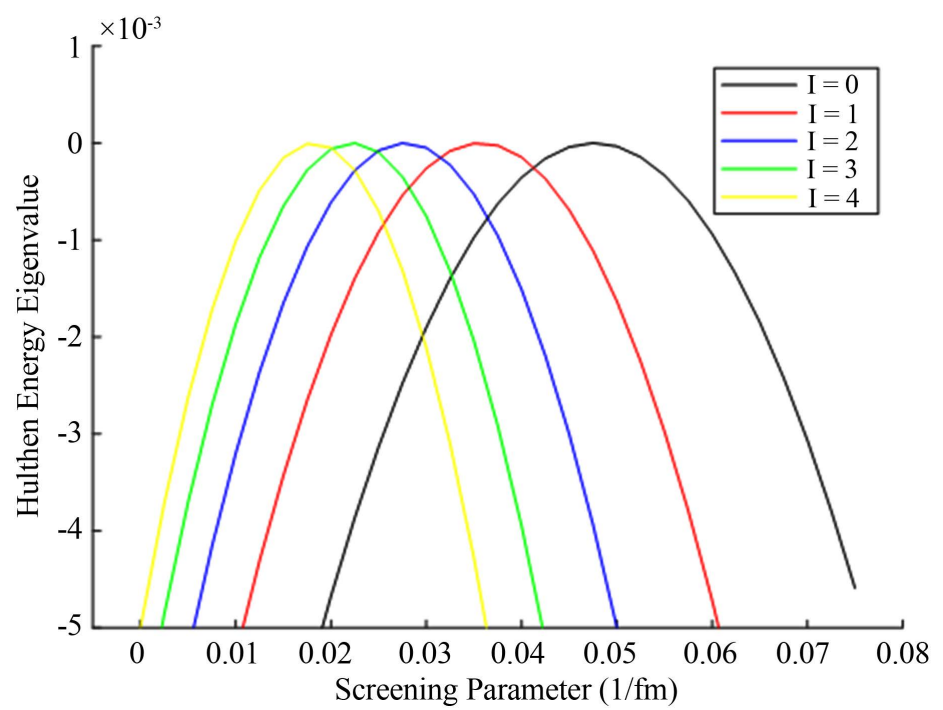

Figure 6. Shifting of energy eigenvalue with increasing Azimuthal quantum number with NU Method. 
Hulthen potential calculated using the NU method. The plot is considered for $n=5$, and $l=0,1,2,3,4$, etc., with the atomic unit. The shifting of energy eigenvalue towards origin is observed. Moreover, the sharpness of the plot is also observed with increased azimuthal quantum numbers. The energy eigenvalue is charge independent, Equation (16), and depends upon screening parameters and quantum numbers. The increasing with the azimuthal quantum number and decreasing screening parameters value the shifting of the energy eigenvalue peak is slow.

The energy eigenvalue for Hulthen potential is calculated in Equation (16) with the help of the NU method and this equation is similar to the energy eigenvalue obtained by Ikhdair and Sever in 2008. Both equations are similar but in our calculation, one extra term with screening parameters is added.

\subsection{Energy Eigenvalue of Hulthen Potential Calculated Using AIM with Screening Parameters}

The representation in Figure 8 below has the same nature as Figure 7 but the nature for $l=0$, quite different observed in Equation (16) or above graph. The calculated value is also different from each other because the Equations (22) and (16) are different from each other, that is Equation (22) has one term $\left(-\frac{\delta}{2}\right)$ less term Equation (16). This term affects the energy eigenvalue calculated from the NU and AIM method, and. More clearly the energy eigenvalue calculated using the NU method is less than that of the energy eigenvalue calculated from AIM.

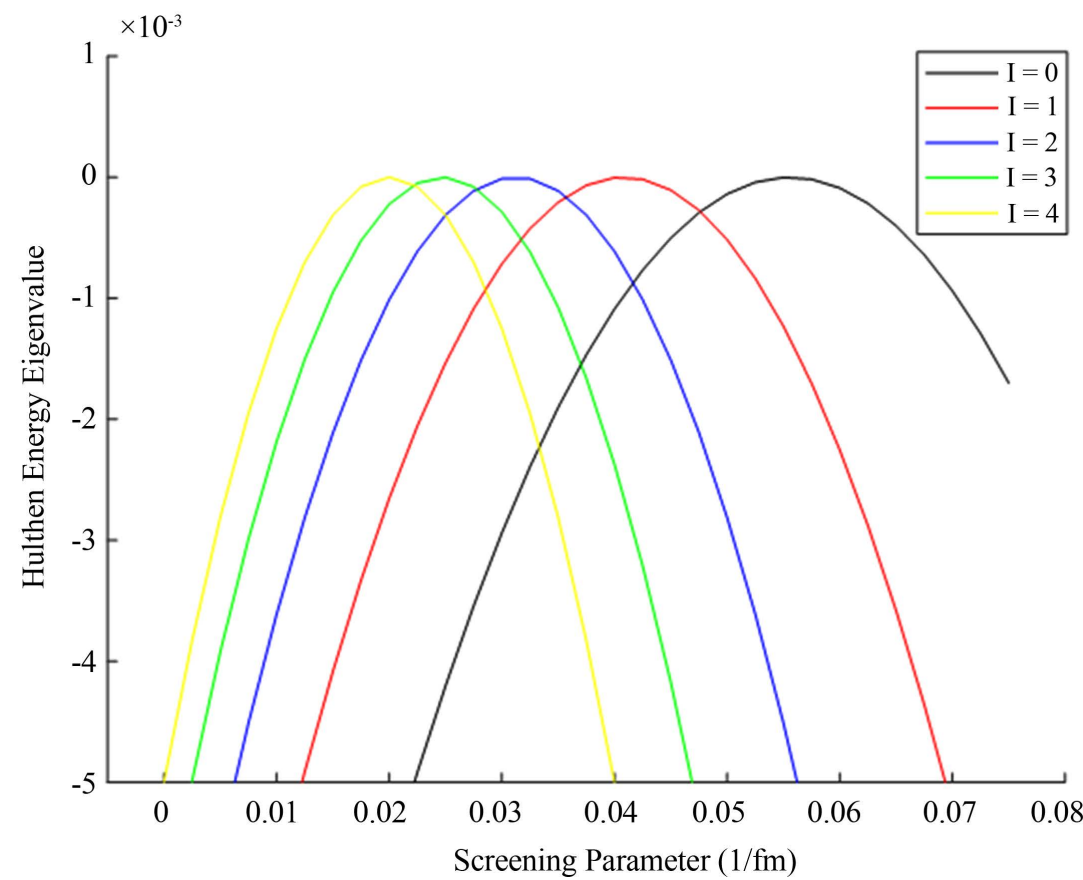

Figure 7. Shifting of energy eigenvalue with increasing Azimuthal quantum number with AIM Method. 


\subsection{Comparison of Energy Eigenvalue Calculated from NU and AIM for Hulthen Potential}

The given Figure 8 below shows the difference of energy eigenvalue calculated using the NU and AIM method for Hulthen Potential. Nature is the same but the numerical value is different due to the presence of $-\frac{\delta}{2}$ in the energy eigenvalue equation calculated with the NU method.
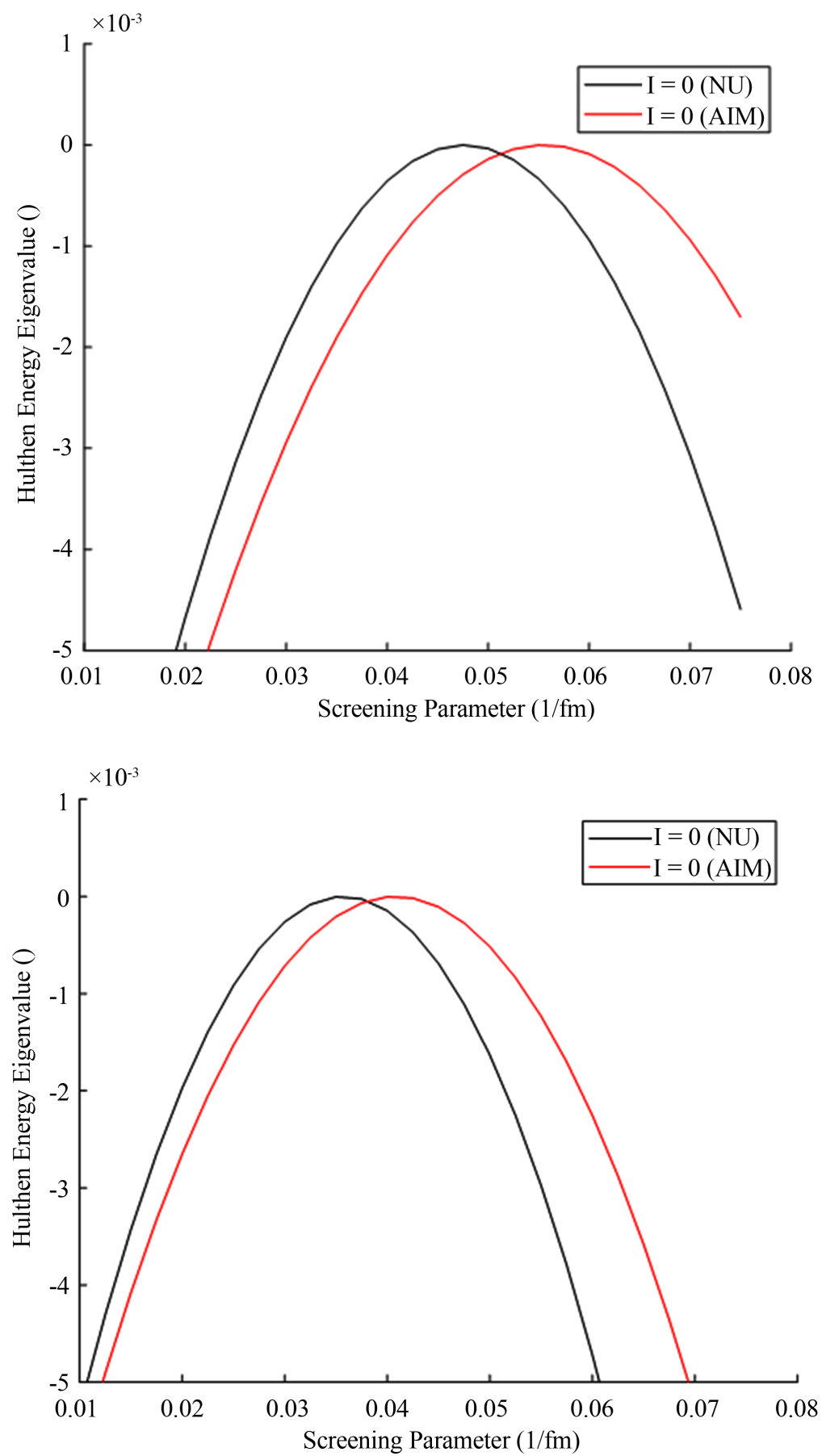

Figure 8. Comparison of energy eigenvalue calculated using NU and AIM method for $l=0$ and $l=1$. 


\section{Conclusion}

The calculation of Energy eigenvalue used the NU method for Hulthen potential with $-\frac{\delta}{2}$. The extra term in Equation (16) shows that the third term of energy eigenvalue is not related to quantum number as the first and second terms of the equation. Therefore, this term does not affect with quantum number of the other two-term. The energy eigenvalue for Hulthen potential is parabolic. The calculation of Energy eigenvalue using the AIM method for Hulthen potential has one less term $-\frac{\delta}{2}$ as shown in Equation (22). Therefore, the energy eigenvalue calculated using AIM is higher than the energy eigenvalue calculated using NU. The third term of Equation (16) is independent of quantum number. Moreover, both equations are charge independent and only depend upon quantum numbers and screening parameters.

\section{Acknowledgements}

We would like to thank all the members of the Department of Physics, Tri-Chandra Multiple Campus, Kathmandu-44600, Tribhuvan University and Department of physics, Patan Multiple Campus, Lalitpur-44700, Robotic Academy of Nepal, Innovative Ghar Nepal, Lalitpur-44700, and National Research Council Nepal for their kind support during this work.

\section{Conflicts of Interest}

The authors declare no conflicts of interest regarding the publication of this paper.

\section{References}

[1] Singh, S.P. (2013) Solving Hydrogen Atom Problem Using Spherical Polar Coordinates: A Qualitative Study. European Journal of Physics Education, 10, 21 p.

[2] Gu, X.Y., Zhang, M. and Sun, J.Q. (2010) Energy Spectra of the Generalized Hulthen Potential. Chinese Journal of Physics, 48, 222-229.

[3] Okon, I.B. and Popoola, O. (2015) Bound-State Solution of Schrodinger Equation with Hulthen Plus Generalized Exponential Coulomb Potential Using Nikiforov-Uvarov Method. International Journal of Recent Advances in Physics, 4, 1-12. https://doi.org/10.14810/ijrap.2015.4301

[4] Fleischer, W. and Soff, G. (1984) Bound State Solutions of the Klein-Gordon Equation for Strong Potentials. Zeitschrift für Naturforschung A, 39a, 703-719. https://doi.org/10.1515/zna-1984-0801

[5] Molaee, Z., Bahar, M.K., Yasuk, F. and Hassanabadi, H. (2013) Solutions of the Duffin-Kemmer-Petiau Equation in the Presence of Hulthén Potential in (1+2) Dimensions for Unity Spin Particles using the Asymptotic Iteration Method. Chinese Physics B, 22, Article ID: 060306. https://doi.org/10.1088/1674-1056/22/6/060306

[6] Bayrak, O. and Boztosun, I. (2007) Bound State Solutions of the Hulthén Potential by Using the Asymptotic Iteration Method. Physica Scripta, 76, 92-96. https://doi.org/10.1088/0031-8949/76/1/016 
[7] Okon, I.B., Popoola, O. and Isonguyo, C.N. (2017) Approximate Solutions of Schrodinger Equation with Some Diatomic Molecular Interactions Using Nikiforov-Uvarov Method. Advances in High Energy Physics, 2017, Article ID: 9671816. https://doi.org/10.1155/2017/9671816

[8] Szego, G. (1939) Orthogonal Polynomials. American Mathematical Society, New York.

[9] Nikiforov, A.V. and Uvarov, V.B. (1988) Special Functions of Mathematical Physics. Birkhauser, Boston. https://doi.org/10.1007/978-1-4757-1595-8

[10] Karayer, H., Demirhan, D. and Büyükkılıç, F. (2015) Extension of Nikiforov-Uvarov Method for the Solution of Heun Equation. Journal of Mathematical Physics, 56, Article ID: 063504. https://arxiv.org/pdf/1504.03518.pdf https://doi.org/10.1063/1.4922601

[11] Radulescu, V. (2008) Rodrigues-Type Formulae for Hermite and Laguerre Polynomials. Analele Stiintifice ale Universitatii Ovidius Constanta, Seria Matematica, 16, 109-116.

[12] Shakil, M., Kibria, B.M.G. and Singh, J.N. (2010) A New Family of Distributions Based on the Generalized Pearson Differential Equation with Some Applications. Austrian Journal of Statistics, 39, 259-278. https://doi.org/10.17713/ajs.v39i3.248

[13] Rajabi, A.A. and Hamzavi, M. (2013) A New Coulomb Ring-Shaped Potential via Generalized Parametric Nikiforov-Uvarov Method. Journal of Theoretical and Applied Physics, 7, Article No. 17.

[14] Bayrak, O. and Boztosun, I. (2007) An Alternative Accurate Solution of the Exponential Cosine Screened Coulomb Potential. International Journal of Modern Physics $C, 18,1443-1451$. https://doi.org/10.1142/S0129183107011455

[15] Gulyamov, A.G. (2015) System of Potential Barriers in Nanostructures. World Journal of Condensed Matter Physics, 5, 60-65.

https://doi.org/10.4236/wjcmp.2015.52009

[16] Thanh, L.D. (2018) Streaming Potential and Zeta Potential Measurements in Porous Rocks. Journal of Geoscience and Environment Protection, 6, 89-100. https://doi.org/10.4236/gep.2018.611007

[17] Rajbongshi, H. and Singh, N.N. (2013) Generation of Exactly Solvable Potentials of position-dependent Mass Schrödinger Equation from Hulthen Potential. Journal of Modern Physics, 4, 1540-1545. https://doi.org/10.4236/jmp.2013.411189

[18] Yoon, J.H., and Yun, Y. (2000) Solution of the S-Wave for Generalized Hulthen Potential by Asymptotic Iteration Method. Journal of the Korean Physical Society, 37, 73-75.

[19] Stanek, J. (2011) Approximate Analytical Solutions for Arbitrary 1-State of the Hulthén Potential with an Improved Approximation of the Centrifugal Term. Central European Journal of Chemistry, 9, 737-742.

https://doi.org/10.2478/s11532-011-0050-6

[20] Elviyanti, I.L., Pratiwi, B.N., Suparmi, A. and Cari, C. (2018) The Application of Minimal Length in Klein-Gordon Equation with Hulthen Potential Using Asymptotic Iteration Method. Advance in Mathematical Physics, 2018, Article ID: 9658679. https://doi.org/10.1155/2018/9658679

[21] Ikhdair, S.M. and Sever, R. (2007) Approximate Eigenvalue and Eigenfunction Solutions for the Generalized Hulthen Potential with Any Angular Momentum. Journal of Mathematical Chemistry, 42, 461-471. https://doi.org/10.1007/s10910-006-9115-8

[22] Gonul, B., Ozer, O., Cancelik, Y. and Kocak, M. (2018) Hamiltonian Hierarchy and 
the Hulthen Potential. Physics Letters A, 275, 238-243.

https://arxiv.org/pdf/nucl-th/0106002.pdf

[23] Sous, A.J. (2008) The Asymptotic Iteration Method for the Eigenenergies of the Complex Potential $V(x)=\gamma x^{4}+i \beta x^{3}+i \alpha x$. Turkey Journal of Physics, 32, 123-131.

[24] Ismail, M.E.H. and Saad, N. (2020) The Asymptotic Iteration Method Revisited. Journal of Mathematical Physics, 61, Article ID: 033501.

https://arxiv.org/pdf/2003.06730.pdf https://doi.org/10.1063/1.5117143

[25] Agboola, D. (2010) Dirac-Hulthén Problem with Position-Dependent Mass in D-Dimensions. arXiv:1011.2368v1. https://arxiv.org/pdf/1011.2368.pdf

[26] Berkdemir, C., Berkdemir, A., and Han, J. (2006) Bound State Solutions of the Schrodinger Equation for Modified Kratzer's Molecular Potential. Chemical Physics Letters, 417, 326-329. https://doi.org/10.1016/j.cplett.2005.10.039

[27] Bayrak, O., Kocak, G. and Boztosun, I. (2006) Any l-State Solutions of the Hulthén Potential by the Asymptotic Iteration Method. Journal of Physics A: Mathematical and General, 39, 11521-11529. http://arxiv.org/abs/math-ph/0609010v1 